\title{
Familial Primary Hypomagnesemia
}

National Cancer Institute

\section{Source}

National Cancer Institute. Familial Primary Hypomagnesemia. NCI Thesaurus. Code C123263.

A hereditary disorder that leads to a selective defect in renal or intestinal magnesium absorption, resulting in a low serum magnesium concentration. 